\title{
Nanosatellite experiments to enable future space-based QKD missions
}

\author{
Robert Bedington ${ }^{* *} \mathbb{D}$, Xueliang Bai ${ }^{1}$, Edward Truong-Cao ${ }^{1}$, Yue Chuan Tan ${ }^{1}$, Kadir Durak' , \\ Aitor Villar Zafra', James A Grieve', Daniel KL Oi² and Alexander Ling ${ }^{1,3}$
}

"Correspondence:

r.bedington@nus.edu.sg

${ }^{1}$ Centre for Quantum Technology, National University of Singapore, 3, Science Drive 2, Singapore, 117543, Singapore

Full list of author information is available at the end of the article

\begin{abstract}
We present a programme for establishing the space worthiness of highly-miniaturised, polarisation-entangled, photon pair sources using CubeSat nanosatellites. Once demonstrated, the photon pair sources can be deployed on more advanced satellites that are equipped with optical links to establish a global space-based quantum key distribution network. In doing so, this work will also bring experimental tests of the overlap between quantum and relativistic regimes closer to realisation.
\end{abstract}

\section{Keywords: QKD; SPDC; CubeSat; SPEQS}

\section{Introduction}

Improvements in quantum computers are increasing the threat to conventional public key encryption. This is driving the development of new "quantum-safe" encryption techniques [1]. Entanglement-based quantum key distribution (QKD) is one of the possible solutions, providing security that is traceable to fundamental correlations between photons, and reducing the number of trusted components when compared with other systems. A global, space-based network for distributing entangled photons will enable strong encryption keys to be delivered securely between any two points on Earth.

Entanglement-based QKD is a mature technology. It has been demonstrated on multiple occasions and is the most established technological application of entanglement. Its range limit however is similar to more commonplace QKD schemes (such as the preparesend-measure approach in the original proposal by Bennett and Brassard [2]). City-sized QKD networks utilising either optical fibre or free-space optics are possible, but the losses within fibres and atmospheric turbulence near the ground restrict the practical range to about a hundred kilometres. To operate on continental and global scales, it is anticipated that future quantum networks would be similar to conventional data networks and employ both fibre-based solutions (quantum-repeater-equipped) and links with optical quantum communication satellites.

Despite much discussion and many theoretical studies, getting relevant quantum technology into space has been hampered by the cost of traditional space development. At the time of writing only one conventional-sized, QKD satellite has been launched, the Chinese QUESS (QUantum Experiments at Space Scale) mission [3]. To accelerate devel- 


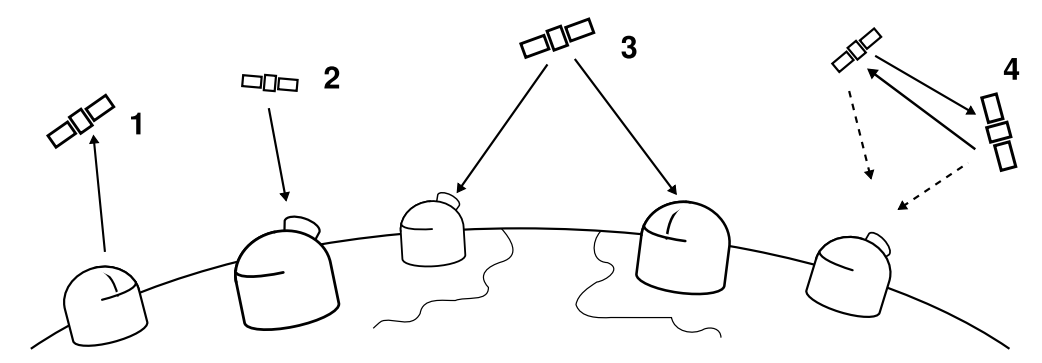

Figure 1 Scenarios for QKD in space. Possible satellite-based QKD implementations. 1. Ground-to-space, where the photon source is on the ground and the satellite only carries detectors. 2. Space-to-ground, where the satellite carries a source and detectors. 3. A platform that can beam down to two ground stations simultaneously. 4. Inter-satellite QKD which could be the building block for a long baseline test of quantum correlations. To enable configurations 2-4 with Bell violation-type measurements, a source of entangled photons in space must be demonstrated.

opment we are seizing on a revolution in the space industry where the use of very small spacecraft known as nanosatellites is enabling access to space for a large number of organisations with only modest funding. Recent years have seen an explosion of interest in nanosatellites, particularly CubeSats [4], where low costs and fast development times are challenging more traditional platforms for applications.

It is our goal to develop a source of entangled photons that is sufficiently bright for spacebased QKD, but with a physical and resource footprint that is compatible with CubeSats. This paper presents an overview of our work towards this goal.

\section{Space-based QKD}

To share quantum encryption keys between space and ground the quantum key (photon) source can be located on the ground or in space [5] (see Figure 1). We (like QUESS) have chosen to focus on having the source in space from which photons are beamed to telescopes on the ground or other spacecraft. This will allow for versatility in QKD experiments (and other experiments [6]) - for example in Figure 1, three of the four QKD configuration options require a space-based source. Beaming photons from space-to-ground also has the advantage that the optical transmission path is much less affected by atmospheric turbulence (because the atmosphere is only significantly thick near the surface of the Earth) so optical link losses are lower [7], although groups have proposed various ground-to-space missions [8-11].

If scenarios 1 or 2 are used to share keys between two ground stations then it is required that the satellites be trusted as secure key exchanging nodes. However, for scenario 3 (such as the SpaceQUEST mission concept [12]) entanglement-based QKD removes that requirement for trust since if one photon in each pair of photons is beamed to Alice (at one ground station) and the other of the pair beamed to Bob (who is at the other ground station) then only Alice and Bob will have knowledge of the polarisations and the key generated is private to them. They will be able to test this quantum exclusivity by performing a Bell violation test [13]. This does of course require ground stations that are concurrently within the field-of-view of the satellite, and since both photons in a pair are travelling along high-loss paths the probability of both photons in a pair reaching their ground stations is very small and thus key generation rates would be very slow. 


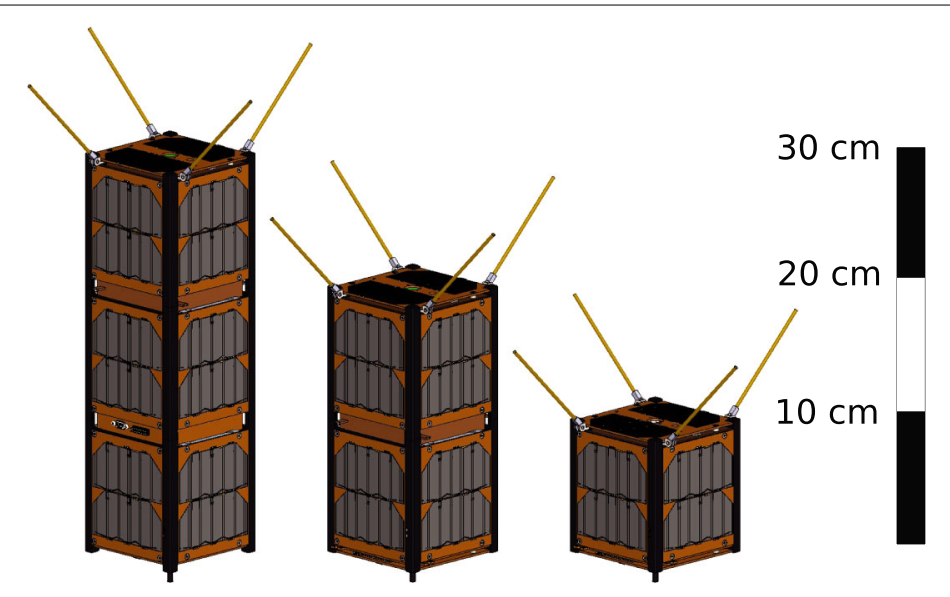

Figure $23 \mathrm{U}, 2 \mathrm{U}$ and $1 \mathrm{U}$ CubeSats. Isometric CAD drawings of $3 \mathrm{U}, 2 \mathrm{U}$ and $1 \mathrm{U}$ CubeSats respectively (based on ISIS structure and GomSpace Gom-X platform).

For most practical purposes though, it is likely that a key exchanging node satellite could be a workable solution. Although they rely on non-quantum security assurances a spacebased node is difficult to access via side-channels. For example, the reported side-channel attacks on terrestrial QKD systems [14] are difficult to implement when targeting a fastflying node where communications can only be achieved over a solid angle of tens of pico steradians.

\section{CubeSat platform}

We are targeting our initial satellite technology developments for use on CubeSats. These use a standardised nanosatellite specification backed by a large and growing industry of compliant subsystems and launch services all of which greatly facilitate access to space.

The smallest typical free-flying CubeSat is a $10 \mathrm{~cm}$ cube and is a fully-functional nanosatellite (i.e. it contains miniaturised solar panels, batteries, onboard computer, radio transceiver as well as a miniature technical or scientific payload). The CubeSat architecture allows the basic cube design (1U) to be extended into larger sized (cuboid) spacecraft measured as multiples of $U(2 \mathrm{U}, 3 \mathrm{U}, 6 \mathrm{U}$ etc., see Figure 2). Depending on launcher and solar panel configurations each $1 \mathrm{U}$ might allow for 1-3 $\mathrm{kg}$ of spacecraft and 1-3 Watts of power.

Since the launch costs are a fraction of the cost of those for traditional spacecraft, CubeSats have become very popular with amateur researchers, university research groups and technology companies seeking a market niche. They are now also gaining popularity with older, well-established space industry players. To keep the hardware budgets small they are typically built from commercial-off-the-shelf (COTS) components rather than expensive, scarce and outdated space-grade parts. Additionally they are often launched into orbits with an orbital life of a few years or even months (although some CubeSats, like Delfi-C3 [15], have been operational now for many years). Space worthiness of COTS components can be assessed by their use in previous CubeSat missions and by carrying out targeted testing.

CubeSats typically reach orbit by ride-sharing with larger satellite launches [16] or are carried as cargo to the International Space Station (ISS) from where they are deployed into 


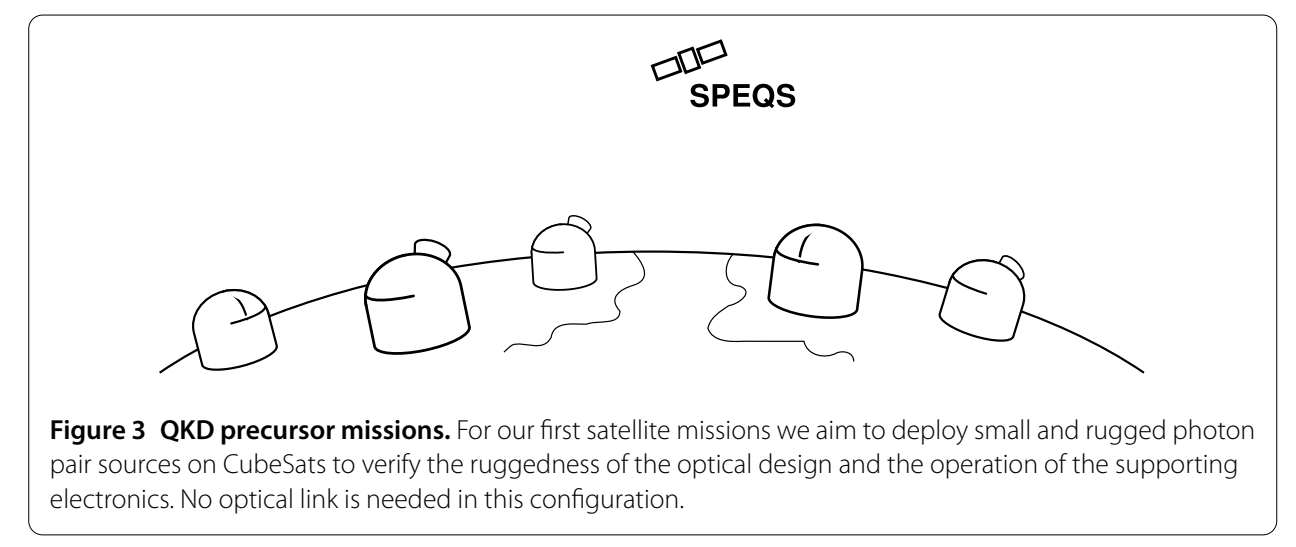

orbit [17]. This "piggy-backing" helps make launch costs affordable to university groups. As with traditional satellites, there is a further level of savings available where each CubeSat can be used to host multiple miniature research payloads. A drawback of this approach is the low duty-cycle for experiment time as the limited power on a CubeSat results in restricted operation time for each payload. Despite the limitations, we propose that it is ultimately feasible to produce pairs of entangled photons on board a CubeSat and to use these pairs to produce a shared quantum key with a ground station or another satellite. The Canadian NanoQEY mission concept meanwhile proposes an alternative ground-tospace (scenario 1 in Figure 1) approach to performing QKD with CubeSats.

Since CubeSats can be built and launched relatively inexpensively and with rapid development cycles, we have chosen to first develop and demonstrate the photon source, before attempting to beam photons to the ground. For the initial technology demonstration missions the photon pair source and the detection apparatus (Bell State analyser) will all be located inside the satellite (Figure 3). Such missions test the ability of the source to survive the vibrations and shocks of launch and investigate how it performs and ages with the ionising radiation, vacuum and thermally demanding conditions of low Earth orbit.

\section{Entangled photon sources for space}

For an optimal space-based QKD source the goal is to maximise key-rate and source longevity, within practical mission constraints. This means producing large rates of highentanglement-quality photon pairs in a compact, robust package. A large number of entangled photon sources based on various physical mechanisms have been reported in the literature, but most of them are incompatible with nanosatellites due to size, weight and power (SWAP) requirements.

Accordingly, we identified sources based on Spontaneous Parametric Downconversion (SPDC) as the most promising. In this class only geometries relying on bulk crystals satisfy all the conditions needed for brightness, quality and ruggedisation. Table 1 shows a comparison of SPDC sources discussed in published literature.

To fit in a CubeSat, the size requirement is critical. For a $1 \mathrm{U}$ CubeSat an experimental payload would typically be contained in a volume that is approximately $10 \mathrm{~cm} \times 10 \mathrm{~cm} \times$ $3 \mathrm{~cm}$. The overall mass of such an instrument should not exceed $500 \mathrm{~g}$, while the continuous (peak) power consumption should be below $2.0 \mathrm{~W}(2.5 \mathrm{~W})$.

Collinear SPDC geometries are favourable because non-collinear sources require a more complex (and less robust) arrangement to achieve a compatible form factor. SPDC sources 
Table 1 Comparison of published results of various SPDC sources

\begin{tabular}{|c|c|c|c|c|}
\hline Paper & Type & $\begin{array}{l}\text { Brightness } \\
\text { pairs } / \mathrm{s} / \mathrm{mm} / \mathrm{mW}\end{array}$ & Collection efficiency $\%$ & Notes \\
\hline Kwiat et al. [18] & $\|$ & 3.3 & & \\
\hline Kwiat et al. [19] & । & 119 & 5 & \\
\hline Fiorentino [20] & $\|$ & 82 & & \\
\hline Fedrizzi et al. [21] & $\|$ & 2,600 & 32 & \\
\hline Trojek and Weinfurter [22] & । & 2,310 & & \\
\hline Trojek and Weinfurter [22] & । & 1,688 & 39 & \\
\hline Rangarajan et al. [23] & । & 11,200 & & BiBo crystal \\
\hline Steinlechner et al. [24] & । & 102,000 & 19 & $10 \mu \mathrm{W}$ pump \\
\hline Da Cunha Pereira et al. [25] & $\|$ & 268 & 42 & $*$ \\
\hline Dixon et al. [26] & $\|$ & 300 & 50 & $*$ \\
\hline Steinlechner et al. [27] & 0 & 50,000 & 45 & \\
\hline Steinlechner et al. [27] & $\|$ & 400 & 45 & \\
\hline Septriani et al. [28] & । & 1,875 & 30 & * \\
\hline
\end{tabular}

The collection efficiency (ratio of detected pairs to single photons) of a source is an important parameter, but one that is not always quoted in papers. Entries marked * indicate sources that produce correlated photon pairs that are not entangled in polarisation.

based on collinear geometries typically utilise quasi-phase-matching conditions. Quasiphase-matching, however, often involves the use of periodically-poled crystals that require precise (within $0.1^{\circ} \mathrm{C}$ ) temperature stabilisation. Satellites in orbit experience cyclical fluctuations of temperature as they pass in and out of the Earth's shadow, but the use of temperature stabilisation (especially with thermo-electric coolers) is challenging on CubeSats because of power and weight restrictions.

Two collinear geometries were considered: critical phase matching with Type-I $\beta$ Barium Borate (BBO) crystals [22] and a non-critical phase matching approach using Type-II periodically poled potassium titanyl phosphate (PPKTP) crystals [27]. PPKTP sources are attractive due to the higher nonlinear coefficients, better tolerances against temperature drift and the possibility of longer crystals (with fewer compensation crystals). BBO sources however have the advantage of using much cheaper crystals, as well as the ability to manufacture crystals with large apertures, making alignment and collection of downconverted light much simpler [28]. To operate with PPKTP crystals would require beam shaping optics that could not be squeezed into the smallest of the CubeSat form-factors. After considering the advantages and disadvantages of the two designs, the BBO-based source was selected for our current devices.

\section{Small photon entangling quantum system (SPEQS)}

The entangled photon pair sources being produced, SPEQS (Small Photon Entangling Quantum Systems), are being developed in iterative cycles that include regular on-orbit testing in CubeSats, as outlined in Table 2. The aim is to gradually raise the technology readiness level of the photon pair sources. The initial iterations developed the miniaturised electronics and ruggedised optics. These led onto on-orbit tests of a correlated photon pair source (these photons are not entangled in polarisation but may have entanglement in other degrees of freedom, e.g. time-frequency).

\subsection{SPEQS-CS}

To address first the challenges of miniaturisation and space-proofing SPEQS-CS, a 1Ucompatible, correlated photon source (with integrated polarisation analyser), has been 
Table 2 Milestones leading to on-orbit QKD demonstrations

\begin{tabular}{|c|c|c|c|}
\hline Year & Milestone & Mission & Reference \\
\hline 2012 & Basic miniature SPDC source & High altitude balloon test & [29] \\
\hline 2013 & Correlated SPDC pair source & High altitude balloon test & [30] \\
\hline 2014 & $\begin{array}{l}\text { Space-qualified, correlated, SPDC } \\
\text { source survives rocket explosion }\end{array}$ & GomSpace GomX-2 CubeSat & [31] \\
\hline 2015 & $\begin{array}{l}\text { Space-qualified, correlated, } \\
\text { SPDC source in low earth orbit }\end{array}$ & NUS Galassia CubeSat & [32] \\
\hline 2017 & $\begin{array}{l}\text { Demonstration of entangled } \\
\text { photon pair source in space }\end{array}$ & CQTSpooQy-1 Cubesat & [33] \\
\hline 2018 & $\begin{array}{l}\text { Demonstration of high-brightness } \\
\text { entangled photon pair source in space }\end{array}$ & CQTSpooQy-2 Cubesat & [34] \\
\hline
\end{tabular}

These missions test the space-worthiness of the devices in-situ and do not involve beaming photons outside the spacecraft. Future missions (at the time of writing) are italicised.

developed. By adopting this compact format the number of flight opportunities for testing it is maximised as it can fly as a guest payload on the CubeSats of third party organisations. Although the room for optical elements is very limited, the platform is an effective solution for proving the functionality of the control electronics, and the space worthiness of the components and the mechanical assembly techniques.

A key challenge for the detection of single photons with avalanche photodiodes (APDs) is maintaining a constant detection efficiency. This is typically achieved by cooling the detectors, e.g. by using thermo-electric coolers, however these require more power than typical CubeSats can supply. By monitoring the pulse height of detection events however, the bias voltage of the APD can be continually adjusted so that its detection efficiency can be maintained in spite of changing temperatures [35].

Another challenge associated with detection is the effect of space radiation on the APDs. Accumulated radiation damage increases the rate of dark counts (thermally generated noise). This noise can be reduced by cooling the detectors but our radiation tests indicate that we should be able to perform our proof of concept demonstrations without cooling. The liquid crystal polarisation rotator performance was found to be relatively insensitive to ionising space radiation [36]. This was also true for the rest of the COTS electronics.

A key test of a SPEQS-CS was performed on a helium balloon in Switzerland [30]. The balloon reached an altitude of $35.5 \mathrm{~km}$ and experienced rapid temperature swings and shock loads yet our device was shown to perform nominally throughout.

A later copy was integrated onto the GomSpace GomX-2 satellite. This CubeSat was prepared for deployment from the ISS but its journey to space was cut short when the rocket it was launched on exploded shortly after lift off. Remarkably the CubeSat was recovered intact from a nearby beach. The satellite was subsequently activated and the SPEQS instrument was found to be fully operational [31]. Another variant of a SPEQS-CS was supplied and integrated onto the NUS Galassia CubeSat [37] which was successfully launched in December 2015. Subsequent demonstration confirmed its status as a spacecapable non-classical light source [32].

\subsection{SPEQS-1}

A polarisation-entangled version of SPEQS (SPEQS-1) is currently being developed. It will be hosted by a dedicated CubeSat (SpooQy-1) being built at CQT and targeted for launch around the end of 2017. The key challenge upgrading from SPEQS-CS to SPEQS-1 
Table 3 SPEQS-CS specifications (GomX-2 version) and design envelopes for upcoming devices

\begin{tabular}{llll}
\hline & SPEQS-CS [31] & SPEQS-1 & SPEQS-2 \\
\hline Brightness & 3,000 & 3,000 & $1,000,000$ \\
Visibility & $96 \%$ & $96 \%$ & $96 \%$ \\
Efficiency & $1 \%$ & $1 \%$ & $20 \%$ \\
Power (peak) & $2.5 \mathrm{~W}$ & $2.5 \mathrm{~W}$ & $10 \mathrm{~W}$ \\
Mass & $0.3 \mathrm{~kg}$ & $0.8 \mathrm{~kg}$ & $1 \mathrm{~kg}$ \\
Volume & $0.25 \mathrm{U}$ & $0.5 \mathrm{U}$ & $0.8 \mathrm{U}$ \\
\hline
\end{tabular}

Brightness refers to the number of detected photon pairs per second per milliwatt. Visibility is a measure of the quality of the photon pairs produced and efficiency refers to percentage of coincident pairs to single photons detected.

\begin{tabular}{|lll|}
\hline 1: laser diode & 10: dichroic mirror & 18,18': pinhole baffle \\
2: focussing lens & 11: BBO3\&4 (spatial-compensator) & $19,19^{\prime}:$ avalanche photodetector \\
3: fluorescence filter & 12: YVO4 (temporal- compensator) & \\
4: half-wave plate & 13: dichroic mirror & \\
5: pinhole & $14,14^{\prime}:$ liquid crystal (as a polariser) \\
6: prism pair & $15,15^{\prime}:$ PBS \\
7: YVO4 (pre-compensator) & $16,16^{\prime}:$ focussing lens \\
8: BBO1\&2 & $17,17^{\prime}:$ interference filter \\
9: long-pass filter & & \\
\hline
\end{tabular}

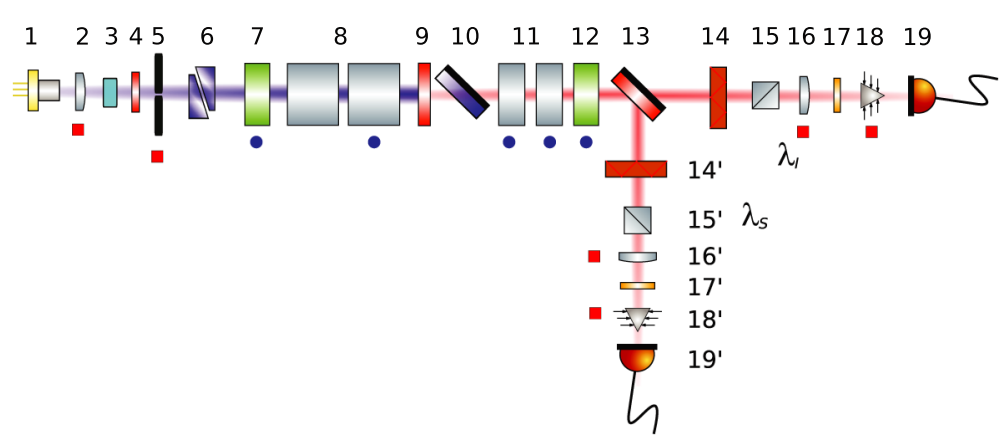

Figure 4 SPEQS-2 optical layout. SPEQS-1 omits the components marked with square red dots, SPEQS-CS additionally omits the components marked with circular blue dots.

is optical alignment. To achieve polarisation entanglement a greater number of crystals are required and their alignment tolerances (approximately $100 \mu \mathrm{rad}$ ) are more demanding.

\subsection{SPEQS-2}

While the SPEQS-1 sources demonstrate the entangled pair production and validation principles in orbit, the 2nd generation devices (SPEQS-2) are being developed to increase the production rate of entangled pairs. The new sources should be sufficiently bright for use in space-to-ground QKD [7]. The target performance specifications are shown in Table 3 and the optical layouts in Figure 4.

Key challenges for the SPEQS-2 development include increasing the APD detection rate to 5 million spontaneous counts per second, managing the extra heat produced by the more powerful pump laser and faster detectors, and keeping the increased number of optical components in alignment [38]. The SPEQS-2 instrument is planned to be launched on the SpooQy-2 spacecraft.

The nominal SpooQySat [39] design is shown in Figure 5 and will be 3U CubeSat with body mounted solar panels, largely-based on the GomSpace GomX platform. 


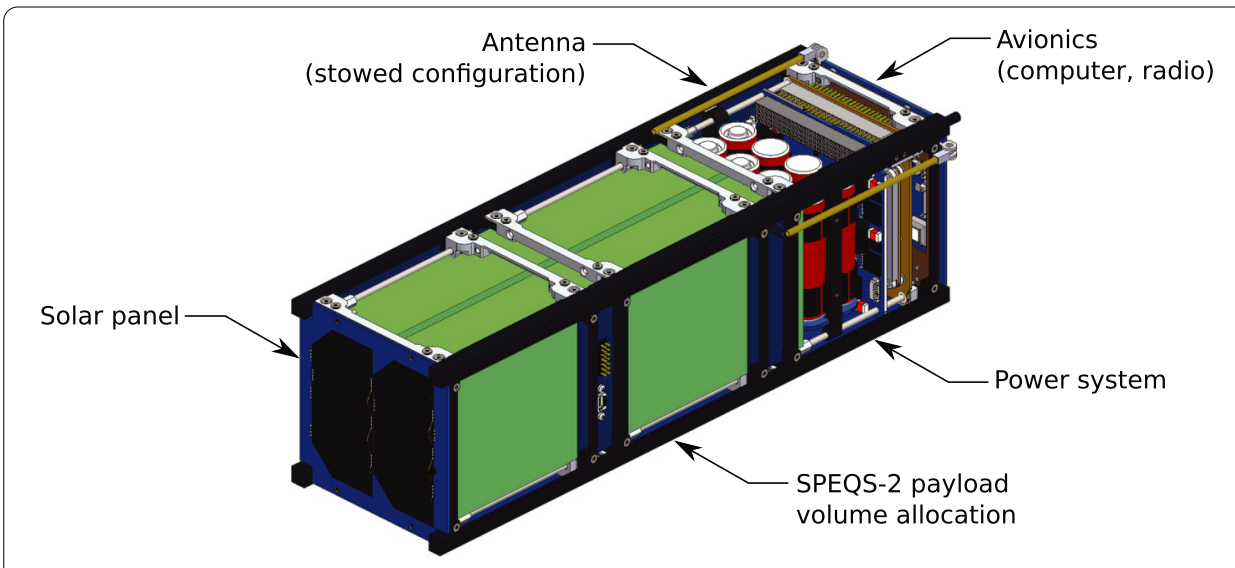

Figure 5 CAD rendering of SpooQy-2 design (with most of the solar panels removed). The green blocks represent the volumes allocated to two SPEQS-2 payloads, allowing redundancy or two design variants of SPEQS to be tested.

If deployed into orbit from the ISS (400 km altitude, 51.65 degree inclination) we estimate it to be able to perform about 90 minutes of SPEQS-2 experiments per day. This could be increased if we use more than just one ground station to downlink the data but is more fundamentally limited by the power generation capabilities of the solar cells (about $6 \mathrm{Wh}$ per day). A $3 \mathrm{U}$ CubeSat in an ISS orbit may have a lifetime of 6-12 months before its orbit degrades; other, longer orbital lifetime options are being investigated.

\section{Outlook and conclusions}

The first practical QKD tests using our SPEQS sources will require measuring one of the photons in the pair on the satellite and beaming the other of the pair down to an optical receiver on Earth or on another satellite. This is a considerably more complex challenge than the current SpooQySat missions. Encouragingly, the technologies for this to be achieved are already existing or in development. Such technologies include high precision satellite position knowledge through GNSS (Global Navigation Satellite System) and laser ranging, high precision ADCS (Attitude Determination and Control Subsystem) [40], optical beam steering [41], transmission optics [42] and optical ground stations [43]. CubeSats have already been built for conventional laser communications [44] and advanced platforms that might one day enable this at the single-photon level are also under development [45].

The rapid progress in experimental quantum optics over the past two decades has been possible because the financial barrier to participation in the scientific work has been modest. As quantum technology matures and its deployment into novel environments begin to take place, the rate of innovation and experimentation may begin to slow down if the financial costs make participation difficult.

Nanosatellite platforms may be key to getting space access for a larger number of university groups, and this in turn, will spur work on getting experiments ready for small spacecraft. We believe the quantum technology community who are interested in space missions cannot afford to overlook the potential of nanosatellites. 
Space-QUEST: Space QUantum Entanglement for Space ExperimenTs

COTS: Commercial Off The Shelf

ISIS: Innovative Solutions In Space

ISS: International Space Station

NanoQEY: Nano Quantum Encryption

SWAP: Size Weight And Power

SPDC: Spontaneous Parametric Down Conversion

BBO: $\beta$-Barium Borate

PPKTP: Periodically Poled Potassium Titanyl Phosphate

SPEQS: Small Photon Entangling Quantum System

SPEQS-CS: SPEQS Correlated Source

NUS: National University of Singapore

CQT: Centre for Quantum Technologies

APD: Avalanche Photo Diode

YVO4: Yttrium Orthovanadate

GNSS: Global Navigation Satellite System

ADCS: Attitude Determination and Control Subsystem

\section{Competing interests}

The authors declare that they have no competing interests.

\section{Authors' contributions}

The concept of using nanosatellites for quantum optics experiments was developed jointly by DO and AL. The other authors are involved either in the production of the quantum light source or the design and assembly of the nanosatellite. All authors read and approved the final manuscript.

\section{Author details}

${ }^{1}$ Centre for Quantum Technology, National University of Singapore, 3, Science Drive 2, Singapore, 117543, Singapore. ${ }^{2}$ SUPA Department of Physics, University of Strathclyde, 107 Rottenrow East, Glasgow, G4 ONG, UK. ${ }^{3}$ Department of Physics, National University of Singapore, 2, Science Drive 3, Singapore, 117551, Singapore.

\section{Acknowledgements}

The Singapore-based team is supported by the Singapore National Research Foundation under its Competitive Research Programme (CRP12-2013-02). D. Oi acknowledges the Scottish Quantum Information Network (QUISCO) and the EU FP7 CONNECT2SEA project "Development of Quantum Technologies for Space Applications".

\section{Received: 13 July 2016 Accepted: 5 October 2016 Published online: 18 October 2016}

\section{References}

1. Campagna M, Chen L, Dagdelen Ö, Ding J, Fernick JK, Gisin N, Hayford D, Jennewein T, Lütkenhaus N, Mosca M, Neill B, Pecen M, Perlner R, Ribordy G, Schanck JM, Stebila D, Walenta N, Whyte W, Zhang Z. Quantum safe cryptography and security. vol. 8. 2015. p. 1-63.

2. Bennett CH, Brassard G. Quantum cryptography: public key distribution and coin tossing. In: Proc IEEE Int Conf Comput Syst Signal Process. vol. 1. 1984. p. 175-9.

3. Gibney E. Chinese satellite is one giant step for the quantum Internet. Nature. 2016;535(7613):478-9. doi:10.1038/535478a.

4. Swartwout M. The first one hundred CubeSats: a statistical look. J Small Satell. 2013;2(2):213-33.

5. Jennewein T, Higgins B. The quantum space race. Phys World. 2013;26(3):52-6. doi:10.1088/2058-7058/26/03/37.

6. Rideout D, Jennewein T, Amelino-Camelia G, Demarie TF, Higgins BL, Kempf A, Kent A, Laflamme R, Ma X, Mann RB, Martín-Martínez E, Menicucci NC, Moffat J, Simon C, Sorkin R, Smolin L, Terno DR. Fundamental quantum optics experiments conceivable with satellites - reaching relativistic distances and velocities. Class Quantum Gravity. 2012;29(22):224011. doi:10.1088/0264-9381/29/22/224011. arXiv:1206.4949.

7. Bourgoin J-P, Meyer-Scott E, Higgins BL, Helou B, Erven C, Hübel H, Kumar B, Hudson D, D'Souza I, Girard R, Laflamme R, Jennewein T. A comprehensive design and performance analysis of low Earth orbit satellite quantum communication. New J Phys. 2013;15(2):023006. doi:10.1088/1367-2630/15/2/023006. arXiv:1211.2733.

8. Scheidl T, Wille E, Ursin R. Quantum optics experiments using the international space station: a proposal. New J Phys. 2013;15(4):043008.

9. Jennewein T, Bourgoin JP, Higgins B, Holloway C, Meyer-Scott E, Erven C, Heim B, Yan Z, Hübel H, Weihs G, Choi E, D'Souza I, Hudson D, Laflamme R. QEYSSAT: a mission proposal for a quantum receiver in space. In: Hasan ZU, Hemmer PR, Lee H, Santori CM, editors. Proc SPIE. vol. 8997. 2014. p. 89970. doi:10.1117/12.2041693.

10. Jennewein T, Grant C, Choi E, Pugh C, Holloway C, Bourgoin J, Hakima H, Higgins B, Zee R. The NanoQEY mission: ground to space quantum key and entanglement distribution using a nanosatellite. In: Gruneisen MT, Dusek M, Rarity JG, Lewis KL, Hollins RC, Merlet TJ, Toet A, editors. Proc SPIE. vol. 9254. 2014. p. 925402. doi:10.1117/12.2067548.

11. Vallone G, Bacco D, Dequal D, Gaiarin S, Luceri V, Bianco G, Villoresi P. Experimental satellite quantum communications. Phys Rev Lett. 2015;115(4):040502. doi:10.1103/PhysRevLett.115.040502. arXiv:1406.4051.

12. Perdigues Armengol JM, Furch B, de Matos CJ, Minster O, Cacciapuoti L, Pfennigbauer M, Aspelmeyer M, Jennewein T, Ursin R, Schmitt-Manderbach T, Baister G, Rarity J, Leeb W, Barbieri C, Weinfurter H, Zeilinger A. Quantum communications at ESA: towards a space experiment on the ISS. Acta Astronaut. 2008;63(1-4):165-78 doi:10.1016/j.actaastro.2007.12.039

13. Ekert AK. Quantum cryptography based on Bell's theorem. Phys Rev Lett 1991;67:661-3. doi:10.1103/PhysRevLett.67.661. arXiv:0911.4171v2. 
14. Gerhardt I, Liu Q, Lamas-Linares A, Skaar J, Kurtsiefer C, Makarov V. Full-field implementation of a perfect eavesdropper on a quantum cryptography system. Nat Commun. 2011;2(2027):349. doi:10.1038/ncomms1348. arXiv:1011.0105.

15. Bouwmeester J, Aalbers GT, Ubbels WJ. Preliminary mission results and project evaluation of the delfi-c3 nano-satellite. Technical report. TU Delft; 2008.

16. Space Flight Services. http://www.spaceflightindustries.com/. Accessed: 2015-06-30.

17. Nanoracks. http://nanoracks.com/. Accessed: 2015-06-30.

18. Kwiat PG, Mattle K, Weinfurter H, Zeilinger A, Sergienko AV, Shih YH. New high-intensity source of polarization-entangled photon pairs. Phys Rev Lett. 1995;75(24):4337-41. doi:10.1103/PhysRevLett.75.4337.

19. Kwiat PG, Waks E, White AG, Appelbaum I, Eberhard PH. Ultrabright source of polarization-entangled photons. Phys Rev A. 1999;60(2):773-6. doi:10.1103/PhysRevA.60.R773.

20. Fiorentino M, Kuklewicz C, Wong F. Source of polarization entanglement in a single periodically poled KTiOPO4 crystal with overlapping emission cones. Opt Express. 2005;13(1):127-35. doi:10.1364/OPEX.13.000127.

21. Fedrizzi A, Herbst T, Poppe A, Jennewein T, Zeilinger A. A wavelength-tunable fiber-coupled source of narrowband entangled photons. Opt Express. 2007;15(23):15377. doi:10.1364/OE.15.015377. arXiv:0708.0813.

22. Trojek $\mathrm{P}$, Weinfurter $\mathrm{H}$. Collinear source of polarization-entangled photon pairs at nondegenerate wavelengths. Appl Phys Lett. 2008;92:211103.

23. Rangarajan R, Goggin M, Kwiat P. Optimizing type-I polarization-entangled photons. Opt Express. 2009;17(21):18920-33. doi:10.1364/OE.17.018920. arXiv:1001.4182.

24. Steinlechner F, Ramelow S, Jofre M, Gilaberte M, Jennewein T, Torres JP, Mitchell MW, Pruneri V. Phase-stable source of polarization-entangled photons in a linear double-pass configuration. Opt Express. 2013;21(10):11943. doi:10.1364/OE.21.011943.

25. Da Cunha Pereira M, Becerra FE, Glebov BL, Fan J, Nam SW, Migdall A. Demonstrating highly symmetric single-mode, single-photon heralding efficiency in spontaneous parametric downconversion. Opt Lett. 2013;38(10):1609-11. doi:10.1364/OL.38.001609.

26. Dixon PB, Rosenberg D, Stelmakh V, Grein ME, Bennink RS, Dauler EA, Kerman AJ, Molnar RJ, Wong FNC. Heralding efficiency and correlated-mode coupling of near-IR fiber-coupled photon pairs. Phys Rev A. 2014;90(4):043804 doi:10.1103/PhysRevA.90.043804. arXiv:1407.8487v1.

27. Steinlechner F, Gilaberte M, Jofre M, Scheidl T, Torres JP, Pruneri V, Ursin R. Efficient heralding of polarization-entangled photons from type-0 and type-II spontaneous parametric downconversion in periodically poled KTiOPO4. J Opt Soc Am B. 2014;31 (9):2068-76. doi:10.1364/JOSAB.31.002068.

28. Septriani B, Grieve JA, Durak K, Ling A. Thick-crystal regime in photon pair sources. Opt. 2016;3(3):347. doi:10.1364/OPTICA.3.000347. arXiv:1511.04159.

29. Morong W, Ling A, Oi D. Space-based quantum network. Opt Photonics News. 2012;23:42-9.

30. Tang Z, Chandrasekara R, Sean YY, Cheng C, Wildfeuer C, Ling A. Near-space flight of a correlated photon system. Sci Rep. 2014;4:6366. doi:10.1038/srep06366.

31. Tang Z, Chandrasekara R, Tan YC, Cheng C, Durak K, Ling A. The photon pair source that survived a rocket explosion. Sci Rep. 2016;6:25603. doi:10.1038/srep25603. arXiv:1512.08834.

32. Tang Z, Chandrasekara R, Tan YC, Cheng C, Sha L, Hiang GC, Oi D, Ling A. Generation and analysis of correlated pairs of photons on board a nanosatellite. Rev Phys Appl. 2016;5:054022. arXiv:1603.06659.

33. Bedington R, Zhongkan T, Chandrasekara R, Cheng C, Chuan TY, Durak K, Zafra AV, Truong-cao E, Ling A, Oi D. Small photon entangling quantum systems (SPEQS) enabling space-based quantum key distribution. In: Int Astronaut Congr. Jerusalem. 2015. p. 1-7.

34. Durak K, Villar A, Septriani B, Chandrasekara R, Tang Z, Bedington R, Ling A. The next iteration of the small photon entangling quantum system (SPEQS-2.0). In: Hasan ZU, Hemmer PR, Lee H, Migdall AL, editors. Proc SPIE. 2016. p. 976209. doi:10.1117/12.2212078.

35. Tan YC, Chandrasekara R, Cheng C, Ling A. Silicon avalanche photodiode operation and lifetime analysis for small satellites. Opt Express. 2013;21(14):16946-54. doi:10.1364/OE.21.016946. arXiv:1306.6773v1.

36. Tan YC, Chandrasekara R, Cheng C, Ling A. Radiation tolerance of opto-electronic components proposed for space-based quantum key distribution. J Mod Opt 2015;62:1709-12. doi:10.1080/09500340.2015.1046519.

37. Sha L, Koenraad M, Seng SW, Hiang GC, Ling A. Galassia system and mission. In: Small Satell Conf. 2014. p. SSC14-XI-2.

38. Chandrasekara R, Tan Z, Tan YC, Cheng C, Septriani B, Durak K, Grieve JA, Ling A. Deploying quantum light sources on nanosatellites I: lessons and perspectives on the optical system. In: Meyers RE, Shih Y, Deacon KS, editors. Proc SPIE. 2015. p. 96150. doi:10.1117/12.2196245. arXiv:1508.07085.

39. Bedington R, Truong-Cao E, Chuan TY, Cheng C, Durak K, Grieve JA, Larsen J, Oi D, Ling A. Deploying quantum light sources on nanosatellites II: lessons and perspectives on CubeSat spacecraft. In: Proc SPIE. 2015. p. 1-10. doi:10.1117/12.2199037. arXiv:1508.07074.

40. Johnston-Lemke B, Sarda K, Grant CC, Zee RE. Arc-minute attitude stability on a nanosatellite: enabling stellar photometry on the smallest scale. In: Proc 25th Annu Small Satell Conf. 2011. p. 1-13.

41. Kingsbury RW, Nguyen T, Riesing K, Cahoy K. Fast-steering solutions for cubesat-scale optical communications. In: Int Conf Sp Opt. Tenerife. 2014. p. 1-6.

42. Kingsbury RW, Caplan DO, Cahoy KL. Implementation and validation of a CubeSat laser transmitter. In: Hemmati H, Boroson DM, editors. Proc SPIE. vol. 9739. 2016. p. 973905. doi:10.1117/12.2217990.

43. Carrasco-Casado A, Kunimori H, Takenaka H, Kubo-Oka T, Akioka M, Fuse T, Koyama Y, Kolev D, Munemasa Y, Toyoshima M. LEO-to-ground polarization measurements aiming for space QKD using Small Optical TrAnsponder (SOTA). Opt Express. 2016;24(11):12254. doi:10.1364/OE.24.012254.

44. Janson SW, Welle RP, Rose TS, Rowen DW, Hinkley DA, Hardy BS, Lumondiere DL, Maul GA, Werner NI. The NASA optical communication and sensors demonstration program: preflight up- date. In: 29th Annu AIAA/USU Conf Small Satell. 2015.

45. Li J, Clark C, Post M, Vick A, Pearson D, Lee D, Macleod D. Practical strategies to stabilize a nanosatellite platform with a space camera and integrated mechanical parts. In: 65th Int Astronaut Congr. Toronto. 2014. 